\title{
Closeout Report Department of Energy Grant DE-FG02 95ER40931 Advanced Map Methods for the Description of Particle Beam Dynamics
}

\author{
Martin Berz and Kyoko Makino, Principal Investigators \\ Center for Beam Theory and Dynamical Systems, MSU
}

\begin{abstract}
The above grant was active at Michigan State University from 1994 until 2007. We summarize and document the various activities and key output under the grant, including degrees awarded to graduate students at MSU and through the VUBeam program sponsored by the grant, the books, publications and reports produced, the meetings organized, and the presentations given.
\end{abstract}




\section{Contents}

I Degrees Awarded 3

II Meetings Organized 4

III Presentations 5

A Inivited Talks by PI Berz . . . . . . . . . . . . . . . 5

B Contributed Talks, Seminars, Colloquia by PI Berz . . . . . . . . . . . 11

C Inivited Talks by PI Makino . . . . . . . . . . . . . . . . . . 13

D Contributed Talks, Seminars, Colloquia by PI Makino . . . . . . . . . . 16

$\begin{array}{ll}\text { IV Courses at Summer Schools etc. } & 20\end{array}$

$\begin{array}{llr}\text { V Publications } & \mathbf{2 1}\end{array}$

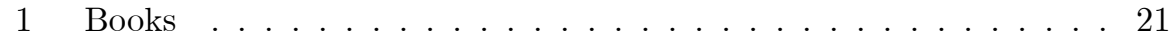

2 Ph.D. Dissertations . . . . . . . . . . . . . . . 21

3 Publications ......................... 21

$\begin{array}{lll}\text { VI Research Bibliography } & 22\end{array}$ 


\section{Degrees Awarded}

1. Johannes Grote, Ph.D. (Defense May 2008)

2. Alexey Poklonskiy, Ph.D. (Defense May 2008)

3. Stephen Weathersby, Ph.D. 2007

4. Pavel Snopok, Ph.D. 2007

5. Xiaosong Geng, M.Sc. 2007 (VUBeam)

6. Salma Al-Rasheed, M.Sc. 2007 (VUBeam)

7. Mahuya Sengupta, M.Sc. 2006 (VUBeam)

8. Shashikant Manikonda, Ph.D. 2006

9. Andrew Steere, M.Sc. 2005 (VUBeam)

10. Carlos Maidana, M.Sc. 2004 (VUBeam)

11. Reiko Taki, Rijken, Japan, M.Sc. 2003 (VUBeam)

12. Mohamad Nasr, King Saud University, Saudi Arabia, M.Sc. 2003 (VUBeam)

13. Ralf Toenjes, M.Sc. 2002

14. Jason Ong, M.Sc. 2002 (VUBeam)

15. Mandi Meidlinger, M.Sc. 2002 (VUBeam)

16. David Meidlinger, M.Sc. 2002 (VUBeam)

17. Bela Erdelyi, Ph.D. 2001

18. Jens Hoefkens, Ph.D. 2001

19. Khodr Shamseddine, Ph.D. 1999

20. Jens von Bergmann, M.Sc. 1999

21. Michael Lindemann, M.Sc. 1998 (VUBeam)

22. Kyoko Makino, Ph.D. 1998

23. Meng Zhao, M.Sc. 1996

24. Weishi Wan, Ph.D. 1995

25. Georg Hoffstätter, Ph.D. 1994 


\section{Meetings Organized}

1. Chair, 2004 Muon Collider Simulation Workshop, Miami, FL, 2004

2. Co-chair, 2004 Computational Accelerator Conference, St. Petersburg, Russia, July 2004

3. Host and Chair, 2002 Computational Accelerator Physics Conference, East Lansing, October 2002

4. Co-chair, Workshops on Taylor Model Methods 2002, 2003, 2004, 2006

5. Host, 1998 Annual USA Symposium of German National Merit Foundation (Studienstiftung des Deutschen Volkes), East Lansing, October 1998

6. Organizer, Symposium on Computational Differentiation and Verified Methods, SIAM Annual Meeting, Toronto 1998

7. Session Chair, APS Spring Meeting 1998

8. Organizer, Symposium on Interval and Computational Differentiation Techniques, SIAM Annual Meeting, Stanford, July 1997

9. Chair, Second SIAM Conference on Computational Differentiation, Santa Fe, New Mexico, February 1996

10. Co-Organizer, Symposium on Computational Differentiation, SIAM Annual Meeting, Kansas City, May 1996

11. Organizer, COSY User's Meeting, Santa Fe, New Mexico, February 1996

12. Organizer, Symposium on Nonlinear Effects in Accelerators, SIAM Conference on Nonlinear Dynamics, Snowbird, Utah, May 1995

13. Session Chair, Fourth Conference on Charged Particle Optics, Tsukuba, October 1994 


\section{Presentations}

\section{A. Inivited Talks by PI Berz}

1. 07/18/07 "Efficient Taylor Model based Verified ODE and Flow Integration using Differential-Algebraic Methods", 6th International Congress on Industrial and Applied Mathematics, Zurich, Switzerland.

2. 05/28/07 "Rigorous Computation of Topological Entropy in the Plane", SIAM Conference on Dynamical Systems, Snowbird, Utah

3. 12/06/06 "Domain Decomposition for Rigorous Integration of Flows", Fourth International Workshop on Taylor Methods, Boca Raton, FL

4. 10/02/06 "A High-Order Differential Algebraic Vlasov Solver", ICAP 06, 9th International Computational Accelerator Physics Conference, Chamonix Mont-Blanc

5. 08/14/06 "Verified Dependency Free Computation" Summer School on Hierarchy and Symmetry in Mathematical Models, Dortmund

6. 08/12/06 "Verified Solution of Elliptic and Vlasov-type PDEs", International Conference on Applied Mathematics, Plovdiv, Bulgaria

7. 07/12/06 "Existence and Uniqueness of Solutions of ODEs in Nonarchimedean Fields", Ninth International Conference on Non-Archimedean Analysis, Concepcion, Chile

8. 06/19/06 - 06/30/06, "Computational Accelerator Physics", Course at US Particle Accelerator School, Boston (with K. Makino)

9. 06/08/06 "Verified Integration of Large Parameter Spaces", Dynamics, Topology, and Computation, Bedlewo, Poland

10. 02/16/06 "The DOE AARD Theory Program", Presentation to HEPAP Review Panel, Chicago

11. 02/06/06 "Large Emittance Simulations of Muon Dynamics with COSY", Muon Collider Conference, Chicago

12. 09/21/05 "Domain Reduction for Validated Global Optimization with Taylor Models", G005, International Workshop on Global Optimization, Almería, Spain. M. Berz.

13. 09/16/05 "An Accurate High-order Verified Method to Solve the 3D Laplace Equation", International Conference on Systems Theory and Scientific Computation, Malta, invited talk. M. Berz.

14. 09/15/05 "High-order Verified Representation of Poincaré Maps", Int. Conf. Systems Theory and Scientific Computation, Malta

15. 08/25/05 "Taylor Model Integration of ODEs, Poincare Sections, and Long-Term Behavior", Second Scandinavian Workshop on Interval Methods and their Applications, Lyngby, Denmark

16. 08/01/05 "Self-Validated Integration of ODEs - Avoiding the Wrapping Effect", Conference on Differential and Difference Equations and Applications, Melbourne, Fl. 
17. 07/11/05 "Performance of Taylor Model Methods for Validated Integration of ODEs", 17th IMACS World Congress, Scientific Computation, Applied Mathematics and Simulation, Paris, France

18. 07/07/05 "Validated Integration of ODEs and PDEs with Taylor Model-based Tools", Conference on Foundations of Computational Mathematics Conference, Santander, Spain, highlited talk

19. 05/27/05 "An Accurate High-order Validated Method to Solve Boundary Value Problems for the Laplace and Poisson Equations based on Taylor Models", SciCADE05, 2005 International Conference on Scientific Computation and Differential Equations, Nagoya, Japan

20. 12/19/04 "Taylor Model Range Bounding Schemes (QFB, Quadratic Fast Bounder and Validated Global Optimization)", Third International Workshop on Taylor Methods, Miami.

21. 12/16/04 "Introduction to Taylor Model Methods", Third International Workshop on Taylor Methods, Miami, tutorial. M. Berz.

22. 12/15/04 "Principles of COSY Simulations of Collider Rings", 2004 Workshop on Muon Collider Simulation, Miami

23. 10/05/04 "Shrink Wrapping and Preconditioning for Validated Integration", 11th GAMM - IMACS International Symposium on Scientific Computing, Computer Arithmetic, and Validated Numerics, SCAN 2004, Fukuoka, Japan. M. Berz.

24. 09/16/04 "High-Order Dependency Free Range Bounding for Validated Global Optimization", NSF Workshop on Reliable Engineering Computing, Savannah, Georgia

25. 07/19/04 "Higher Order Methods", Fourth Conference on Automatic Differentiation, Chicago, invited tutorial

26. 07/09/04 "Different Forms of Differentiability on $\{\mathrm{L}\}$ evi- $\{\mathrm{C}\}$ ivita Fields and their Consequences", Eighth International Conference on p-adic Analysis, Clermont-Ferrand, France, invited talk

27. 06/29/04 "Recent Advances in Modern Map Methods", Eighth Computational Accelerator Physics Conference, St. Petersburg, Russia, plenary lecture.

28. 04/23/04 "Validated High-Order Methods and Applications to Integration", Fifth International Conference on Applied Mathematics, Fort Lauderdale, invited talk

29. 01/27/04 "COSY High-Order Field Computation", Muon Collaboration Meeting, Riverside, California, invited talk

30. 08/27/03 " Muon Emittance Exchange Workshop, Chicago, invited talk

31. 08/21/03 "Validation of Transfer Maps Using Taylor Models", Physics and Control 2003, St. Petersburg, Russia, plenary talk

32. 08/15/03 "Taylor Model Methods and Applications to Nonlinear Dynamics", Scandinavian Workshop on Interval Methods and Their Applications, Copenhagen, plenary talk

33. 08/19/03 "Global Optimization with Taylor Model Methods", 18th International Symposium on Mathematical Programming, Copenhagen 
34. 08/12/03 "New Applications of Taylor Model Methods", Numerical Analysis and Computer Science 2002, Plovdiv, Bulgaria

35. 05/22/03 "Proving Long-Term Stability with Validated ODE Integrators", Dynamical Systems and Applications 2002, Atlanta

36. 06/08/02 "Map Methods in Cooling Simulations", NUFACT 2002, New York

37. 01/20/02 "Higher Order Convergence", Symposium on Validated Methods, Dagstuhl, Germany

38. 12/16/02 "Introduction to Taylor Model Methods", 2002 Taylor Model Workshop, Miami

39. 11/21/02 "COSY simulations of Ring Coolers", Ring Cooler and Emittance Exchange Workshop, Fermilab

40. 10/23/02 "New Approaches for the Validation of Transfer Maps using RemainderEnhanced Differential Algebra", Sixth Charged Particle Optics Conference, Greenbelt, Maryland

41. 08/12/02 "Self-Validated Integration from the Perspective of Computational Dynamics", 2002 Conference on Foundations of Computational Mathematics, Minneapolis, $\mathrm{MN}$

42. 08/07/02 "Nonlinear Dynamics in Large Particle Accelerators", International Conference on Scientific Computation, Atlanta, GA

43. 06/21/02 "A Cauchy Theory on the Levi-Civita Field", Seventh International Conference on Non-Archimedean Analysis, Nijmegen, Netherlands

44. 06/11/02 "Recent Advances in Self-Validated Methods", World Automation Congress, Orlando, FL

45. 05/30/02 "Recent Advances in the Treatment of the Wrapping Effect", Workshop on Validated Methods for Optimization, Fields Institute, Toronto

46. 05/29/02 "Self-Validated Implementation of Numerics in the Taylor Model Framework", Workshop on Validated Methods for Optimization, Fields Institute, Toronto

47. 05/28/02 "What Taylor Models Are Not", Workshop on Validated Methods for Optimization, Fields Institute, Toronto

48. 05/23/02 "Taylor Model Based Verified Integration for the Volterra Equations and the Lorenz System", SIAM Workshop on Validated Computing, Toronto

49. 05/23/02 "Validated Integration of Asteroid Orbits", SIAM Workshop on Validated Computing, Toronto

50. 05/22/02 "Taylor Models and Their Heuristics for Global Optimization", SIAM Conference on Optimization, Toronto

51. 05/11/02 "Recent Muon Simulation Results with COSY", Neutrino Factory and Muon Collider Collaboration Meeting, Shelter Island, NY

52. 03/09/02 "Nonlinear Effects and 6D Dynamics in Muon Accelerator Scenarios", Muon Collaboration Meeting, Los Angeles, CA 
53. 11/21/01 "Verified Integration under Avoidance of the Wrapping Effect", Symposium on Numerical Integration and its Complexity, Oberwolfach, Germany

54. 10/09/01 "Simulation of Quadrupole Cooling Channel", 2001 Emittance Exhange Workshop, Berkeley, California

55. 09/11/01 "Taylor Model-based Verified Integrators", Workshop on Verified Solution of Differential Equations, Fields Institute, Toronto

56. 08/09/01 "High-Order Verified Methods and Applications to Symplectic Integration", Workshop on the Dynamics of Numerics, Fields Institute, Toronto

57. 08/02/01 "Verified ODE and DAE integration controlling the wrapping effect", SciCaDE 2001, Vancouver

58. 07/16/01 "Recent Advances in Differential Algebraic Methods", Workshop on Nonlinear Beam Dynamics, Snowmass, CO

59. 07/14/01 "Map Methods for the Analysis of Cooling Dynamics", Workshop on Cooling Dynamics, Snowmass, CO

60. 07/13/01 "Normal Form Methods and Optimization for Nonlinear Properties of Cooling Channels", Workshop on Cooling Dynamics, Snowmass, CO

61. 07/03/01 "Verified Solutions of Differential Equations without Wrapping", 2001 International Conference on Applied Mathematics, Pohang, Korea

62. 09/20/00 "Higher Order Verified Methods", SCAN2000 - Interval 2000, Karlsruhe, Germany

63. 08/12/00 "Preservation of Hamiltonian Structure in 3D Curvilinear Dynamics", 2000 Colloquium on Numerical Analysis and Computer Science, Plovdiv, Bulgaria

64. 07/21/00 "Verified Integration of ODEs", WCNA 2000, Catania, Italy

65. 07/10/00 "Verified Integration and Taylor Model Methods in Nonlinear Dynamics", WSES 2000, Vouliagmeni, Greece

66. 07/03/00 "Verified Methods for Control Theory", CAO 2000, Sankt Petersburg, Russia

67. 07/01/00 "Spin Dynamics", 2000 Conference on Beam Dynamics and Optimization, St. Petersburg, Russia

68. 06/21/00 "Towards a Universal Data Type for Scientific Computing", Automatic Differentiation 2000, Nice, selected talk

69. 06/14/00 "Verified Integration of Near-Earth Asteroids", WAC 2000, Maui, HI

70. 06/11/00 "Verified Computational Methods", Symposium and Tutorial on Modern Methods in Numerics, Maui, HI

71. 05/24/00 "Nonlinear Effects in Neutrino Factories", Neutrino Factory Conference, Monterey, CA

72. 05/01/00 "Effects of Fringe Fields on Muon Ring Dynamics, APS Spring Meeting, Long Beach, CA

73. 12/16/99 "Nonlinear Effects in Muon Storage Rings, 1999 Muon Collider and Neutrino Factory Conference, San Francisco, CA 
74. 11/21/99 "Verified Integration of ODEs, Symbolic-Algebraic Methods and Verification, Dagstuhl, Germany

75. 10/01/99 "Fringe Field and Kinematic Effects in Muon Rings, 1999 Muon Collider Collaboration Meeting, Montauk, NY

76. 08/15/99 "Experiences with On-Line Education in Beam Physics", International Internet Education Workshop, Romania,

77. 08/13/99 "New Methods for Verified Integration of ODEs", Eighth International Colloquium for Numerical Analysis, Plovdiv, Bulgaria

78. 05/14/99 "The Taylor Model Method for Verified Integration of ODEs", Symposium on Verified Integration, Atlanta, GA

79. 05/13/99 "Verified Integration in Celestial Mechanics", SIAM Annual Meeting, Atlanta, GA

80. 03/23/99 "The Beam Physics Virtual University Initiative", APS Centennial Meeting, Atlanta, GA

81. 10/29/98 "Verified Integration of Orbits and Flows", Second MICS Workshop on Predictability of Complex Systems, Albuquerque, NM,

82. 07/14/98 "Verified Computational Differentiation through Numerical Integrators", SIAM Annual Meeting, Toronto, Canada

83. 07/07/98 "Verified Integration under avoidance of the Wrapping Effect", 1998 International Conference on Differential Equations, St. Louis, MO

84. 06/30/98 "Nonlinear Beam Dynamics - New Trends", 1998 Conference on Beam Dynamics and Optimization, St. Petersburg, Russia

85. 05/19/98 "Verified Differentiation through ODE solvers", 1998 Computational Differentiation Theory Institute, Argonne, IL

86. 04/21/98 "Recent Advances in Differential Algebraic Methods", APS Spring Meeting, Columbus, $\mathrm{OH}$

87. 12/10/97 "Experiences with International Distance Education", National Research Council / National Academy of Science Panel on Distance Education, Washington, D.C.

88. 10/16/97 "Estimating the Effects of Remainders in Map Methods", 1997 International Conference on Beam Dynamics and Optimization, Dubna (not presented due to visa problems)

89. 07/28/97 "Rigorous Long-Term Stability Estimates in Particle Accelerators", SPIE Symposium on Charged Particle Optics, San Diego, CA

90. 07/17/97 "Taylor Model Methods for the Integration of ODEs", SIAM Annual Meeting, Stanford, CA

91. 05/18/97 "Applications of COSY INFINITY for the Muon Collider", Muon Collaboration Annual Meeting, Orcas Island, WA

92. 05/07/97 "Dependency Elimination and Higher-order Bounding with RDA Methods", 1997 Workshop on Interval Methods, El Paso, TX 
93. 12/09/96 through 12/13/96 "Differential Algebraic Techniques and Applications", Series of four invited lectures, DESY/KFA Symposium on Mathematical Aspects of Accelerator Physics, Bad Honnef, Germany

94. 12/03/96 "From Taylor Series to Taylor Models and Remainder Differential Algebras," Symposium on Nonlinear Effects in Accelerator Physics, Santa Barbara, CA

95. 11/23/96 "Infinitely Small Numbers and Almost Infinitely Large Accelerators", Studienstiftung Annual USA Meeting, Dallas, TX

96. 09/25/96 "Differential Algebras with Remainder and Rigorous Proofs of Long-Term Stability," Fourth Conference on Computational Accelerator Physics, Williamsburg, VA

97. 09/16/96 "Computational Differentiation with Remainder Bounds," International Conference on Modelling and Computing in Physics, Dubna

98. 07/26/96 "Taylor Models, Small Numbers, and Large Accelerators: Proving Long Term Stability of Weakly Nonlinear Systems," SIAM Symposium on Verification Theory, Techniques and Software, Kansas City, KS

99. 07/24/96 "Infinitely Small Numbers and Their Use in Beam Physics," SIAM Annual Meeting, Kansas City, KS

100. 07/16/96 "Rigorous Bounds on Survival Times for Motion around Elliptical Fixed Points ," Second World Congress on Nonlinear Analysis, Athens, Greece

101. 07/04/96 "Rigorous Bounds for Stability Times in Storage Rings," Beam Dynamics and Optimization 1996, St. Petersburg, Russia

102. 05/29/96 "Stability of Weakly Nonlinear Dynamical Systems," Nonlinear Dynamics 96, Springfield, MO

103. 02/13/96 "The Applications of COSY INFINITY to Nonlinear Dynamics," Second SIAM Conference on Computational Differentiation, Santa Fe, NM

104. 02/12/96 "Calculus and Numerics on Levi-Civita Fields," Second SIAM Conference on Computational Differentiation, Santa Fe, NM

105. 07/08/95 "Stability of Weakly Nonlinear Systems," ISSAC Montreal,

106. 07/05/95 "COSY INFINITY and its Use in Beam Physics," ICIAM 95, Hamburg

107. 05/22/95 "Differential Algebraic Methods in Beam Physics," SIAM Conference on Nonlinear Dynamics, Snowbird, UT

108. 02/25/95 "Proving Stability of Complex Dynamical Systems," Conference on Interval Methods, El Paso, TX

109. 10/05/94 "Modern Map Methods," Fourth Charged Particle Optics Conference, Tsukuba, Japan

110. 09/21/94 "Differential Algebraic Formulation of Spin Dynamics," 1994 Conference on Spin Physics, Bloomington, IN

111. 09/13/94 "The Use of Interval Methods for the Estimation of Stability in Particle Accelerators," Workshop on Verified Computation, Karlsruhe, Germany

112. 09/06/94 "Modern Map Methods in Accelerator Physics," Conference on Nonlinear Problems in Accelerators, Arcidosso, Italy 


\section{B. Contributed Talks, Seminars, Colloquia by PI Berz}

1. 09/28/07 "Rigorous Integration of Flows of ODEs", Department of Aerospace Dynamics, University of Milan, Italy

2. 09/26/07 "Rigorous Determination of Bounds on Topological Entropy in the Plane", University of Amsterdam, Netherlands

3. 07/23/07 "Domain Decomposition for the Solution of Flows", Seminar, University of Karlsruhe, Germany

4. 09/21/05 "Domain Reduction for Validated Global Optimization with Taylor Models", G05, International Workshop on Global Optimization, Almería, Spain

5. 08/01/05 "Self-Verified Integration of ODEs - Avoiding the Wrapping Effect", Conference on Differential and Difference Equations and Applications, Melbourne, FL

6. 05/31/05 "Stability Estimates by Validated Global Optimization", KEK, Japan, Seminar

7. 12/16/04 "Introduction to Taylor Model Methods", Third International Workshop on Taylor Methods, Miami, tutorial

8. 10/05/04 "Shrink Wrapping and Preconditioning for Validated Integration", 11th GAMM - IMACS International Symposium on Scientific Computing, Computer Arithmetic, and Validated Numerics, SCAN 2004, Fukuoka, Japan

9. 10/05/04 "High-Order Constraint Satisfaction via Taylor Models", 11th GAMM IMACS International Symposium on Scientific Computing, Computer Arithmetic, and Validated Numerics, SCAN 2004, Fukuoka, Japan

10. 07/05/04 "Asymptotic Behavior of Preconditioned Long-Term Validated Integration", University of Karlsruhe, Seminar

11. 06/25/04 "Validated ODE Integration - Methods and Tools", University of Frankfurt, Seminar

12. 06/23/04 "Advances in Validated ODE Integration", PARA 2004, Copenhagen

13. 05/12/04 "Asymptotic Stability of High-Order Validated Integration", Fields Institute, University of Toronto, Seminar

14. 12/14/03 "Asymptotic Behavior of Validated Integration Algorithms", 2003 Taylor Model Workshop, Miami

15. 09/15/03 "Preconditioning Methods for Validated Integration, University of Toronto, Seminar

16. 12/18/02 "Validated ODE Integration", 2002 Taylor Model Workshop, Miami

17. 12/17/02 "Comparison of Bounding with Taylor Models and Other Modern Tools", 2002 Taylor Model Workshop, Miami

18. 07/30/02 "Ring Cooler Simulations in COSY based on Transfer Maps", Seminar, Fermilab

19. 06/19/02 "Measure Theories on Non-Archimedian Levi-Civita Fields", Contributed Talk, Conference on Non-Archimedean Analysis, Nijmegen, 
20. 06/12/02 "Reconstructive Correction of Large Particle Spectrographs", Seminar, Catania

21. 06/11/02 "Fringe Field Effects in Quadrupole Cooling Channels", Seminar, Fermilab

22. 05/29/02 "Taylor Models - Order of Convergence and the Linear Dominated Bounder", Fields Institute Thematic Program on Numerical and Computational Challenges in Science and Engineering, Validated Methods for Optimization, Toronto

23. 05/23/02 "Methods of Proving Chaos in Dynamical Systems via Transfer Maps", SIAM Workshop on Validated Computing, Toronto

24. 05/21/02 "Tight Range Enclosures with Taylor Model Methods", SIAM Conference on Optimization, Toronto

25. 03/19/02 "Quadrupole Channel Cooling Simulations", Seminar, Fermilab

26. 01/25/02 "Chaos at Large Accelerators", MSU Campus Theory Seminar

27. 10/12/01 "VUBeam - The Online Master's and Ph.D. program in Beam Physics", Seminar, College of Natural Science, MSU

28. 08/08/01 "Verified ODE solvers", Contributed Talk, Workshop on Dynamics of Numerics, Fields Institute, Toronto

29. 04/12/01 "The MSU VUBeam Initiative and Related Activities", Seminar, Fermilab

30. 02/13/01 "Dependency Reducing Extensions of Interval Methods", Seminar, Sun Microsystems, Palo Alto, CA

31. 10/16/00 "Verified Computational Methods", Colloquium, Kansas State University, Manhattan, KS

32. 09/13/00 "Nonlinear Effects in Muon Storage Rings", Contributed Talk, International Computational Accelerator Physics Conference, Darmstadt, Germany

33. 08/10/00 "Verified Integration of ODEs", Contributed Talk, BIT2000, Lund, Sweden

34. 07/06/00 "Analysis and Computational Methods for the Levi-Civita Field", Contributed Talk, 2000 Conference on Non-Archimedean Analysis, Ioannina, Greece

35. 08/05/99 "Dependency-Free Verified Methods", Seminar, University of Aachen, Germany

36. 07/15/98 "High-Order Verified Methods and Control of Dependency and Wrapping Effect", Contributed Talk, SIAM Annual Meeting, Toronto

37. 04/14/98 "The Influence of Fringe Fields on Particle Dynamics in the LHC", Contributed Talk, Fifth Conference on Charged Particle Optics, Delft, Netherlands

38. 04/14/98 "High-Order Maps and Bounds for Taylor Remainders", Contributed Talk, Fifth Conference on Charged Particle Optics, Delft, Netherlands

39. 04/07/98 "New Verified Methods for High-Performance Applications", Seminar, Mathematics and Computer Science Division, Argonne National Laboratory

40. 04/06/98 "Nonlinear Effects in Accelerators: Fringe Fields, Resonances, and Momentum Compaction and other delights", Seminar, Fermilab 
41. 11/15/97 "Nonlinear Dynamics Simulation of the Muon Collider", Seminar, Fermilab

42. 07/18/97 "Verified Integrators for Maps", Seminar, Stanford Linear Accelerator Center

43. 05/14/97 "New Features in COSY INFINITY", 1997 Particle Accelerator Conference, selected talk

44. 04/17/97 "Experiences with Distance Education in Beam Physics", Seminar, College of Natural Science, MSU

45. 03/25/97 "Nonlinear Map Methods", Seminar, Fermilab

46. 07/30/96 "Remainder Differential Algebras and Their Use in Global Optimization," Seminar, Mathematics and Computer Science Division, ANL, Argonne, IL

47. 05/10/96 "Reconstructive Correction of Aberrations in Spectrographs," Seminar, CEBAF, Newport News, VA

48. 02/16/96 "Remainder Differential Algebras," Contributed Talk, COSY User's Meeting, Santa Fe, NM

49. 09/21/95 "Paradoxes of Theoretical Physics and Modern Logic," Seminar, Olang Summer School, Studienstiftung des Deutschen Volkes

50. 07/06/95 "Problems Associated with a Covariant General Relativistic Treatment of Spin Dynamics," Seminar, DESY Hamburg

51. 10/13/94 "Differential Algebras and Superconvergent Newton Methods," Seminar, Institute of Statistical Mathematics, Tokyo University

52. 10/13/94 "Differential Algebraic Methods in Beam Physics," Seminar, Institute of Statistical Mathematics, Tokyo University

53. 03/11/94 "From Differential Algebras to Remainder Differential Algebras," Seminar, Institute for Nuclear Research, Moscow State University

54. 03/08/94 "Rigorous Proofs for Long-Term Stability of Dynamical Systems," Contributed Talk, Conference on Interval Arithmetic 1994, St. Petersburg

55. 03/02/94 "Rigorous Proofs for Long-Term Stability of Dynamical Systems," Seminar, Mathematics and Computer Science Division, Argonne

\section{Inivited Talks by PI Makino}

1. 06/05/06. "Demonstration of the code COSY INFINITY", DyToComp 2006, International Conference on Dynamics, Topology and Computations, Bedlewo, Poland.

2. 06/04/06. "Taylor Model-based Verified Integration of ODEs", DyToComp 2006, International Conference on Dynamics, Topology and Computations, Bedlewo, Poland.

3. 09/16/05. "Range Bounding with Taylor Models for Global Optimization", 5th WSEAS International Conference on Systems Theory and Scientific Computation, Malta.

4. 08/25/05. "Advances in Global Optimization with Taylor Models", Second Scandinavian Workshop on Interval Methods and their Applications, Lyngby, Denmark. 
5. 07/11/05. "Taylor Model Methods for High Performance Validated Computations", 17th IMACS World Congress, Scientific Computation, Applied Mathematics and Simulation, Paris, France.

6. 07/08/05. "Validated Global Optimization and Stability Estimates", FoCM 2005, Foundations of Computational Mathematics Conference, Santander, Spain.

7. 05/27/05. "Taylor Model-based Validated Integration of ODEs", SciCADE05, 2005 International Conference on Scientific Computation and Differential Equations, Nagoya, Japan.

8. 09/15/04. "Recent Advances in the Validated Integration of ODEs", NSF Workshop on Reliable Engineering Computing, Savannah, GA.

9. 04/22/04. "Range Bounding with Taylor Models - Some Case Studies", WSEAS MATH 2004, Differential Equations: Theory and Applications Symposium, Fort Lauderdale, FL.

10. 01/28/04. "Straight Quadrupole Cooling Channel Simulations", Muon Collaboration Meeting, Riverside, CA.

11. 09/10/03. "Simulation of Muon Ionization Cooling Systems using Transfer Map Method", Illinois Institute of Technology, Chicago, IL.

12. 08/28/03. "Quadrupole Cooling Channel Simulation - Particles from Buncher, Phase Rotator through the Quad-Channel in COSY", Ring Coolers and Emittance Exchange Workshop, Fermilab, IL.

13. 08/26/03. "Simulation of Muon Ionization Cooling Systems using Transfer Map Method", University of Illinois at Urbana-Champaign, Urbana, IL.

14. 08/21/03. "Nonlinear Transfer Map Treatment of Beams through Systems with Absorbing Material", International Conference Physics and Control, Saint Petersburg, Russia.

15. 08/15/03. "High Order Range Bounding Using Taylor Models", 1st Scandinavian Workshop on Interval Methods and Their Applications, Technical University of Denmark, Copenhagen, Denmark.

16. 06/06/03. "Balbekov (Tetra) Ring Simulation Results in COSY", NuFact03, 5th International Workshop on Neutrino Factories \& Superbeams, Columbia University, New York.

17. 01/24/03. "Quad Cooling Channel Simulation", MuCool Meeting, Fermilab, IL.

18. 05/31/02. "Taylor Models - Hand Calculation for a Linear ODE System", Fields Institute, Thematic Programs, Numerical and Computational Challenges in Science and Engineering, Validated Methods for Optimization, Toronto, Canada.

19. 05/28/02. "Taylor Models - Roundoff", Fields Institute, Thematic Programs, Numerical and Computational Challenges in Science and Engineering, Validated Methods for Optimization, Toronto, Canada.

20. 05/23/02. "Taylor Model Based Verified Integration for the Volterra Equations and the Lorenz System", SIAM Workshop on Validated Computing, Toronto, Canada.

21. 05/13/02. "Nonlinear Effects in Quadrupole Cooling Channels", Neutrino Factory and Muon Collider Collaboration Meeting, Shelter Island, NY. 
22. 03/08/02. "COSY Simulation Code", Ring Cooler Workshop, UCLA, Los Angeles, CA.

23. 03/07/02. "Linear/Nonlinear Cooling Theory", Ring Cooler Workshop, UCLA, Los Angeles, CA.

24. 09/13/01. "Verified Integration with Taylor Models - Nonlinear Examples", Fields Institute, Thematic Programs, Numerical and Computational Challenges in Science and Engineering, Validated Methods for ODEs and DAEs, Toronto, Canada.

25. 06/20/01. "Magnet Fringe Fields, Nonlinear Effects, and Compensation in Large Acceptance Rings", 2001 Particle Accelerator Conference, Chicago, IL.

26. 05/15/01. "High-order Transfer Maps and Verification of Stability in Particle Accelerators", Understanding Complex Systems Symposium, University of Illinois at Urbana-Champaign, May 15, 2001.

27. 05/03/01. "Tracking Study on Nonlinear Effects in the 20-GeV Feasibility II Muon Storage Ring", Nu-mu Collaboration Meeting, Fermilab, May 2-3, 2001.

28. 10/10/01. "Cooling Channel Simulation by COSY Infinity", Emittance Exchange Workshop, Lawrence Berkeley National Laboratory, Berkeley, CA.

29. 10/09/01. "Quad Cooling Channel Simulation", Emittance Exchange Workshop, Lawrence Berkeley National Laboratory, Berkeley, CA.

30. 09/19/00. "Advances in Verified Integration of ODEs", SCAN 2000, International Symposium on Scientific Computing, Computer Arithmetic, and Validated Numerics, Karlsruhe, Germany.

31. 08/15/00. "Perturbative Equations of Motion and Differential Operators in Nonplanar Curvilinear Coordinates", 9th International Colloquium on Numerical Analysis and Computer Science with Applications, Plovdiv, Bulgaria.

32. 07/12/00. "Verified Global Optimization with Taylor Model Methods", WSES CSCC-MCP-MCME 2000, Athens, Greece.

33. 07/07/00. "Nonlinear Spin Dynamics", Beam Dynamics Optimization, BDO2000, St. Petersburg, Russia.

34. 07/07/00. "Nonlinear Effects on the Dynamics in Muon Storage Rings", Beam Dynamics Optimization, BDO2000, St. Petersburg, Russia.

35. 07/06/00. "Differential Algebraic Methods for Feedforward Control Theory", 11th IFAC International Workshop, Control Applications of Optimization, CAO2000, St. Petersburg, Russia.

36. 07/05/00. "Verified Control of Near-Earth Asteroid Orbits", CAO2000, St. Petersburg, Russia.

37. 06/14/00. "Verified Integration of Near-Earth Asteroids", World Automation Congress, WAC2000, Maui, Hawaii.

38. 12/14/99. "Effects of Kinematic Correction", Neutrino Factory and Muon Collider Collaboration Meeting, Berkeley, CA.

39. 05/13/99. "Control of the Dependency Problem", SIAM Annual Meeting, Atlanta, Georgia. 
40. 06/30/98. "Rigorous Long-term Stability Estimates", BDO-98, Fifth International Workshop, Beam Dynamics and Optimization, St. Petersburg, Russia.

41. 05/20/98. "Differential Algebraic Methods on Taylor Models", Argonne Theory Institute on Differentiation of Computational Approximations to Functions, Argonne, IL.

42. 04/23/98. "Verified High Order Numerical Integrators Based on Taylor Models", International Conference on Interval Methods and their Application in Global Optimization (INTERVAL'98), Nanjing, China.

43. 04/23/98. "New Methods for High-Dimensional Verified Quadrature", International Conference on Interval Methods and their Application in Global Optimization (INTERVAL'98), Nanjing, China.

44. 07/18/97. "Verified Analysis of Stability in Particle Accelerators", SIAM 45th Anniversary Meeting, Stanford, CA.

45. 05/15/97. "Rigorous Integration of Maps and Long-Term Stability", 1997 Particle Accelerator Conference, Vancouver, BC, Canada.

46. 02/16/96. "Integration through Measured Fields", COSY User's Symposium, Santa Fe, New Mexico.

\section{Contributed Talks, Seminars, Colloquia by PI Makino}

1. July 18, 2007. "High-Order Box Rejection and Pruning for Global Optimization based on Taylor Model Methods", 6th International Congress on Industrial and Applied Mathematics, Zurich, Switzerland, July 16-20, 2007.

2. 12/18/06. "Recent Advances in Taylor Model based Rigorous Global Optimization", 4th International Workshop on Taylor Methods, Boca Raton, Florida.

3. 10/04/06. "COSY INFINITY", ICAP 06, 9th International Computational Accelerator Physics Conference, Chamonix Mont-Blanc, France. Code Demonstration Panel.

4. 10/03/06. "Rigorous Global Optimization for Parameter Estimates and Long-Term Stability Bounds", ICAP 06, 9th International Computational Accelerator Physics Conference, Chamonix Mont-Blanc, France.

5. 09/29/06. "Dependency Reduced High-Order Derivative based Box Rejection for Global Optimization", SCAN' 2006, 12th GAMM - IMACS International Symposion on Scientific Computing, Computer Arithmetic and Validated Numerics, Duisburg, Germany.

6. 06/12-16/06. USPAS course "Computational Accelerator Physics", USPAS, Boston. Lectures.

7. 09/22/05. "Practical Performance of Verified Global Optimization Tools using Taylor Models", G05, International Workshop on Global Optimization, Almería, Spain.

8. 08.01/05. "Validated Flows, Normal Forms, and the Stability of the Tevatron", Conference on Differential and Difference Equations and Applications, Melbourne, FL.

9. 05/31/05. "Validated Integration of ODEs", KEK, Japan. Seminar. 
10. 12/19/04. "Taylor Model Range Bounding Schemes (LDB, Linear Dominated Bounder and QDB, Quadratic Dominated Bounder)", Third International Workshop on Taylor Methods, Miami, FL.

11. 12/18/04. "Preconditioning Methods in the Taylor Model ODE Integrator", Third International Workshop on Taylor Methods, Miami, FL.

12. 10/08/04. "Range Bounding for Global Optimization with Taylor Models", SCAN 2004, 11th GAMM - IMACS International Symposium on Scientific Computing, Computer Arithmetic, and Validated Numerics, Fukuoka, Japan.

13. 10/07/04. "Rigorous Stability Estimates for the Dynamics in the Tevatron via Nekhoroshev-type Estimates based on Normal Form Pseudo Invariants", SCAN 2004, 11th GAMM - IMACS International Symposium on Scientific Computing, Computer Arithmetic, and Validated Numerics, Fukuoka, Japan.

14. 10/05/04. "Shrink Wrapping and Preconditioning for Validated Integration", SCAN 2004, 11th GAMM - IMACS International Symposium on Scientific Computing, Computer Arithmetic, and Validated Numerics, Fukuoka, Japan.

15. 07/09/04. "Computational Implementation and Application of the Arithmetic and Operations of the Levi-Civita Field", 8th International Conference on p-adic Functional Analysis, Clermont-Ferrand, France.

16. 06/30/04. "COSY INFINITY Version 9", Eighth Computational Accelerator Physics Conference, St. Petersburg, Russia.

17. 12/20/03. "High Order Range Bounding Using Taylor Models - LDB and QDB", Taylor Model Symposium, Miami, FL.

18. 08/01/03. "Quadrupole Cooling Channel Simulation - Particles from Buncher, Phase Rotator through the Quad-Channel in COSY", Ring Coolers/Emittance Exchange Meeting, Fermilab, IL.

19. 02/04/03. "Progress on Linear Quadrupole Cooling Channel", Ring Coolers / Emittance Exchange Meeting, Fermilab, IL.

20. 01/28/03. "Progress on Linear Quadrupole Cooling Channel", Ring Coolers / Emittance Exchange Meeting, Fermilab, IL.

21. 12/16/02. "Basics of Taylor Models", Taylor Model Symposium, Miami, FL.

22. 12/16/02. "Taylor Models and Other Polynomial Methods", Taylor Model Symposium, Miami, FL.

23. 12/17/02. "Comparison of Taylor Model Bounding with Other State of the Art Methods", Taylor Model Symposium, Miami, FL.

24. 12/18/02. "COSY's Taylor Model Operations", Taylor Model Symposium, Miami, FL.

25. 12/18/02. "Validated ODE Integration with Taylor Models", Taylor Model Symposium, Miami, FL.

26. 12/20/02. "Taylor Model ODE Solver - An Example", Taylor Model Symposium, Miami, FL.

27. 11/21/02. "COSY Simulations on Muon Beam Ring Cooler by V. Balbekov", RingCoolers and Emittance Exchange Workshop, Fermilab, IL. 
28. 10/23/02. "COSY INFINITY", CPO-6, 2002 International Charged Particle Optics Conference, Greenbelt, Maryland.

29. 10/23/02. "High Order Map Treatment of Cavities and Absorbers with Superimposed Solenoidal Fields", CPO-6, 2002 International Charged Particle Optics Conference, Greenbelt, Maryland.

30. 10/17/02. "New Solenoid Elements in COSY INFINITY", 7th International Computational Accelerator Physics Conference, MSU, East Lansing, MI.

31. 07/30/02. "Balbekov Ring Simulation in COSY (Transfer Maps)", Ring Coolers/Emittance Exchange Meeting, Fermilab, IL.

32. 06/11/02. "Fringe Field Effects in Quadrupole Cooling Channels", Ring Coolers/Emittance Exchange Meeting, Fermilab, IL.

33. 05/29/02. "Taylor Models - Order of Convergence, the Linear Dominated Bounder", Fields Institute, Thematic Programs, Numerical and Computational Challenges in Science and Engineering, Validated Methods for Optimization, Toronto, Canada.

34. 05/21/02. "Tight Range Enclosures with Taylor Model Methods", SIAM Conference on Optimization, Toronto, Canada.

35. 03/19/02. "Quad Channel Cooling Simulation", Ring Coolers / Emittance Exchange Meeting, Fermilab, IL.

36. 09/14/01. "Dependency Free Range Bounding", Fields Institute, Thematic Programs, Numerical and Computational Challenges in Science and Engineering, Validated Methods for ODEs and DAEs, Toronto, Canada.

37. 09/11/01. "Validated ODE Integration of Taylor Models and Examples", Fields Institute, Thematic Programs, Numerical and Computational Challenges in Science and Engineering, Validated Methods for ODEs and DAEs, Toronto, Canada.

38. 08/08/01. "Optimal Control of the Wrapping Effect in Taylor Model based Verified Integration", Fields Institute, Thematic Programs, Numerical and Computational Challenges in Science and Engineering, Dynamics of Numerics, Toronto, Canada.

39. 07/16/01. "Recent Application of COSY to Nonlinear Beam Dynamics Problems", Snowmass 2001, the Future of Particle Physics, Snowmass, Colorado.

40. 07/14/01. "Cooling Channel Simulation based on Map Methods", Snowmass 2001, the Future of Particle Physics, Snowmass, Colorado.

41. 07/03/01. "Verified Solutions of ODEs over Large Domains without Wrapping", $\mathrm{Com}^{2} \mathrm{MaC}-2$, Pohang, Korea.

42. 03/20/01. "Nonlinear Dynamics in Muon Accelerators", University of Illinois at Urbana-Champaign. Seminar.

43. 03/12/01. "Report on BNL Storage Ring Study", Muon Collaboration Meeting.

44. 08/28/00. "Fringe Field Computation and COSY Infinity", KEK, Tsukuba, Japan.

45. 08/10/00. "Verified High Order Range Enclosure of Multivariate Functions", 40th Anniversary of the Journal BIT - Numerical Mathematics, BIT2000, Lund, Sweden.

46. 07/21/00. "Higher Order Verified Inclusions of Multidimensional Systems by Taylor Models", 3rd World Congress of Nonlinear Analysts, WCNA2000, Catania, Italy. 
47. 06/27/00. "Verification of Invertibility and Charting of Constraint Manifolds in Differential Algebraic Equations", 6th IMACS International IMACS Conference on Applications of Computer Algebra, IMACS ACA 2000, St. Petersburg, Russia.

48. 06/27/00. "Differential Algebraic Structures and Verification", IMACS ACA 2000, St. Petersburg, Russia.

49. 06/23/00. "New Applications of Taylor Model Methods", 3rd International Conference on Automatic Differentiation, AD2000, Nice, France.

50. 05/07/98. "Precise and Verified Calculation of High Order Transfer Maps for General Fields", KEK, Tsukuba, Japan.

51. 04/15/98. "COSY Infinity Version 8", Fifth International Conference on Charged Particle Optics, Delft University of Technology, The Netherlands.

52. 04/06/98. "Nonlinear Effects in Rings: Fringe Fields, Resonances, Momentum Compactions, and Other Delights", Fermilab, IL.

53. 02/10/98. "Precise and Verified Calculation of High Order Maps for General Fields", Fermilab, IL.

54. 01/98. "Taylor Models, and Verified Integration of ODEs and Flows", Lecture at USPAS course "Computational Methods in Beam Physics" by Martin Berz, University of Texas at Austin, Austin, Texas.

55. 01/98. "Equation of Motion in Curvilinear Coordinates", Lecture at USPAS course "Computational Methods in Beam Physics" by Martin Berz, University of Texas at Austin, Austin, Texas.

56. 01/98. "Introduction to COSY INFINITY", Lecture at USPAS course "Computational Methods in Beam Physics" by Martin Berz, University of Texas at Austin, Austin, Texas.

57. 07/28/97. "Arbitrary Order Aberrations for Elements Characterized by Measured Fields", SPIE Optical Science, Engineering, and Instrumentation 42nd Annual Meeting, San Diego, CA.

58. 05/08/97. "Efficient Verified Integration Schemes", 1997 Interval Workshop, ACM Symposium on Theory of Computing, El Paso, Texas.

59. 02/20/97. "Introduction to COSY INFINITY", Lecture in video telecast course PHY861 "Introduction to Beam Physics", Michigan State University.

60. 09/25/96. "COSY INFINITY Version 7", 1996 Computational Accelerator Physics Conference, Williamsburg, Virginia.

61. 02/12/96. "Remainder Differential Algebras and Their Applications", Second International Workshop on Computational Differentiation, Santa Fe, New Mexico.

62. 06/95. "Equation of Motion in Particle Optical Coordinates", Lecture at USPAS course "Introduction to Beam Optics" by Martin Berz, University of Washington, Seattle, Washington. 


\section{Courses at Summer Schools etc.}

1. Course on Computational Accelerator Physics, 3 Credits, 2006 US Particle Accelerator School, Boston/Massachusetts

2. Course on Computational Accelerator Physics, 3 Credits, 1998 US Particle Accelerator School, Austin/Texas

3. Course on Non-Archimedean Analysis, Studienstiftung Summer Academy, Olang/Italy 1995

4. Course on Particle Optics, 3 Credits, 1995 US Particle Accelerator School, Seattle/Washington 


\section{Publications}

During the performance period, a variety of publications were produced. These include a total of five books, nine PhD dissertations, 110 refereed publications, 32 nonrefereed publications, and 21 technical reports have been produced.

\section{Books}

1. D. O. Ovsyannikov, M. Berz and K. Makino (Eds.), Computational Accelerator Physics, Elsevier 2006

2. M. Berz, K. Makino (Eds.), Computational Accelerator Physics, IOP Publishing, 2004

3. M. Berz, K. Makino and W. Wan, Introduction to Beam Physics, in progress

4. M. Berz, Modern Map Methods in Particle Beam Physics, Academic Press, 1999

5. M. Berz, C. Bischof, A. Griewank and G. Corliss (Eds.), Computational Differentiation: Techniques, Applications, and Tools, SIAM, Philadelphia, 1996

\section{Ph.D. Dissertations}

1. Stephen Weathersby ${ }^{1}$

2. Pavel Snopok ${ }^{2}$

3. Shashikant Manikonda ${ }^{3}$

4. Bela Erdelyi ${ }^{4}$

5. Jens Hoefkens ${ }^{5}$

6. Khodr Shamseddine ${ }^{6}$

7. Kyoko Makino ${ }^{7}$

8. Weishi $\mathrm{Wan}^{8}$

9. Georg Hoffstätter ${ }^{9}$

\section{Publications}

The publications are listed in the bibliography; items 1-9 are dissertations, 10-119 are refereed publications, 120-151 are non-refereed publications, and 152-172 are technical reports. 


\section{Research Bibliography}

${ }^{1}$ S. Weathersby. Damping Higher Order Modes in the PEP-II B-Factory Storage Ring Collider. PhD thesis, Michigan State University, East Lansing, Michigan, USA, 2007.

${ }^{2}$ P. Snopok. Optimization of Accelerator Parameters Using Normal Form Methods on High-Order Transfer Maps. PhD thesis, Michigan State University, East Lansing, Michigan, USA, 2007.

${ }^{3}$ S. Manikonda. High Order Finite Element Methods to Compute Taylor Transfer Maps. $\mathrm{PhD}$ thesis, Michigan State University, East Lansing, Michigan, USA, 2006.

${ }^{4}$ B. Erdélyi. Symplectic Approximation of Hamiltonian Flows and Accurate Simulation of Fringe Field Effects. PhD thesis, Michigan State University, East Lansing, Michigan, USA, 2001.

${ }^{5}$ J. Hoefkens. Verified Methods for Differential Algebraic Equations. PhD thesis, Michigan State University, East Lansing, Michigan, USA, 2001.

${ }^{6} \mathrm{~K}$. Shamseddine. New Elements of Analysis on the Levi-Civita Field. PhD thesis, Michigan State University, East Lansing, Michigan, USA, 1999. http://bt.pa.msu.edu/cgi-bin/display.pl?name=shamseddinephd.

${ }^{7}$ K. Makino. Rigorous Analysis of Nonlinear Motion in Particle Accelerators. PhD thesis, Michigan State University, East Lansing, Michigan, USA, 1998. Also MSUCL1093.

${ }^{8} \mathrm{~W}$. Wan. Theory and Applications of Arbitrary-Order Achromats. PhD thesis, Michigan State University, East Lansing, Michigan, USA, 1995. also MSUCL-976.

${ }^{9}$ G. H. Hoffstätter. Rigorous bounds on survival times in circular accelerators and efficient computation of fringe-field transfer maps. $\mathrm{PhD}$ thesis, Michigan State University, East Lansing, Michigan, USA, 1994. also DESY 94-242.

${ }^{10}$ J. Grote S. Newhouse, M. Berz and K. Makino. On the estimation of topological entropy on surfaces. Contemporary Mathematics, 469:243-270, 2008.

${ }^{11} \mathrm{~K}$. Shamseddine and M. Berz. Intermediate value theorem for analytic functions on a Levi-Civita field. Bulletin of the Belgian Mathematical Society, 14:1001-1015, 2007.

${ }^{12}$ J. Grote, M. Berz, K. Makino, and S. Newhouse. Taylor model-based enclosure of invariant manifolds for planar diffeomorphisms and applications. ICIAM, 6, 2007.

${ }^{13}$ P. Di Lizia, F. Bernelli Zazzera, and M. Berz. High order integration and sensitivity analysis of multibody systems using differential algebra. AIDAA, 19, 2007.

${ }^{14}$ C. J. Johnstone, M. Berz, and K. Makino. Staging acceleration and cooling in a neutrino factory. Nuclear Instruments and Methods, 558,1:282-291, 2006.

${ }^{15} \mathrm{~K}$. Shamseddine and M. Berz. Generalized power series on a non-Archimedean field. Indagationes Mathematicae, 17,3:457-477, 2006.

${ }^{16}$ K. Makino and M. Berz. Suppression of the wrapping effect by Taylor model- based verified integrators: The single step. International Journal of Pure and Applied Mathematics, 36,2:175-197, 2006.

${ }^{17}$ Alexey Poklonskiy, Dmitri A. Ovsyannikov, Alexander D. Ovsyannikov, David Neuffer, and Martin Berz. Optimization of the buncher and phase rotator for a neutrino factory. SPbSU Messenger (Vestnik), 10,1, 2006. 
${ }^{18} \mathrm{~S}$. L. Manikonda and M. Berz. Multipole expansion solution of the Laplace equation using surface data. Nuclear Instruments and Methods, 558,1:175-183, 2006.

${ }^{19}$ Kyoko Makino, Martin Berz, Youn-Kyung Kim, and Pavel Snopok. Long term stability of large accelerators. ECMI Newsletter, 39, 2006.

${ }^{20}$ J. Grote, M. Berz, and K. Makino. High-order DA methods for the determination of Poincare sections. Nuclear Instruments and Methods, 558,1:106-111, 2006.

${ }^{21}$ Pavel Snopok, Dmitri A. Ovsyannikov, Alexander D. Ovsyannikov, Martin Berz, and Carol Johnstone. Modeling and optimization of muon collider interaction regions. SPbSU Messenger (Vestnik), 10,1, 2006.

${ }^{22}$ K. Makino and M. Berz. Suppression of the wrapping effect by Taylor model- based verified integrators: Long-term stabilization by preconditioning. International Journal of Differential Equations and Applications, 10,4:353-384, 2005.

${ }^{23} \mathrm{M}$. Berz and K. Makino. Suppression of the wrapping effect by Taylor model- based verified integrators: Long-term stabilization by shrink wrapping. International Journal of Differential Equations and Applications, 10,4:385-403, 2005.

${ }^{24}$ M. Berz, K. Makino, and Y.-K. Kim. Long-term stability of the Tevatron by validated global optimization. Nuclear Instruments and Methods, 558:1-10, 2005.

${ }^{25} \mathrm{~K}$. Shamseddine and M. Berz. Analytical properties of power series on nonArchimedean fields. Annales Mathematiques Blaise Pascal, 12:309-329, 2005.

${ }^{26}$ S. L. Manikonda, M. Berz, and K. Makino. High-order verified solution of the 3D Laplace equation. Transactions on Computers, 4-11:1604-1610, 2005.

${ }^{27}$ P. Snopok, M. Berz, K. Makino, and C. Johnstone. Simulation and optimization of the Tevatron accelerator. Lecture Notes on Computational Science and Engineering, 50:199-209, 2005.

${ }^{28}$ J. Grote, K. Makino, and M. Berz. High-order validated representation of Poincaré maps. Transactions on Systems, 4,11:1986-1992, 2005.

${ }^{29}$ A. A. Poklonskiy, D. Neuffer, C. J. Johnstone, M. Berz, K. Makino, D. A. Ovsyannikov, and A. D. Ovsyannikov. Optimizing adiabatic bunchers and phase rotators. Nuclear Instruments and Methods, 558:135-141, 2005.

${ }^{30}$ J. Grote, M. Berz, and K. Makino. High-order representation of Poincare maps. Lecture Notes on Computational Science and Engineering, 50:59-66, 2005.

${ }^{31}$ C. Johnstone, M. Berz, and K. Makino. Optimal staging of acceleration and cooling in a neutrino factory. Nuclear Physics, 149 Suppl.:316-319, 2005.

${ }^{32}$ K. Makino and M. Berz. Range bounding for global optimization with Taylor models. Transactions on Computers, 4,11:1611-1618, 2005.

${ }^{33}$ P. V. Snopok, C. J. Johnstone, M. Berz, D. A. Ovsyannikov, and A. D. Ovsyannikov. Study and optimal correction of a systematic skew quadrupole field in the Tevatron. Nuclear Instruments and Methods, 558:142-146, 2005.

${ }^{34} \mathrm{M}$. Berz and K. Makino. Performance of Taylor model methods for validated integration of ODEs. In Lecture Notes in Computer Science, volume 3732, pages 65-74, 2005. 
${ }^{35}$ S. L. Manikonda and M. Berz. An accurate high-order method to solve the Helmholtz boundary value problem for the 3D Laplace equation. International Journal of Pure and Applied Mathematics, 23,3:365-378, 2005.

${ }^{36} \mathrm{~K}$. Makino and M. Berz. COSY INFINITY version 9. Nuclear Instruments and Methods, 558:346-350, 2005.

${ }^{37} \mathrm{M}$. Berz and K. Makino. New approaches for the validation of transfer maps using remainder-enhanced differential algebra. Nuclear Instruments and Methods A, 519:53-62, 2004.

${ }^{38}$ N. Revol, K. Makino, and M. Berz. Taylor models and floating-point arithmetic: Proof that arithmetic operations are validated in COSY. Journal of Logic and Algebraic Programming, 64/1:135-154, 2004.

${ }^{39}$ B. Erdélyi and M. Berz. Local theory and applications of extended generating functions. International Journal of Pure and Applied Mathematics, 11,3:241-282, 2004.

${ }^{40}$ D. Errede, K. Makino, M. Berz, C. J. Johnstone, and A. van Ginneken. Stochastic processes in muon ionization cooling. Nuclear Instruments and Methods A, 519:466471, 2004.

${ }^{41}$ K. Makino, M. Berz, and Y.-K. Kim. Range bounding with Taylor models - some case studies. Transactions on Mathematics, 3,1:137-145, 2004.

${ }^{42}$ C. J. Johnstone, M. Berz, D. Errede, and K. Makino. Muon beam ionization cooling in a linear quadrupole channel. Nuclear Instruments and Methods A, 519:472-482, 2004.

${ }^{43} \mathrm{~K}$. Makino and M. Berz. Tetra cooler ring simulation in COSY INFINITY. In Neutrino Factories and Superbeams, volume 721, page 418. AIP Conference Proceedings, 2004.

${ }^{44}$ K. Makino, M. Berz, D. Errede, and C. J. Johnstone. High order map treatment of superimposed cavities, absorbers, and magnetic multipole and solenoid fields. Nuclear Instruments and Methods A, 519:162-174, 2004.

${ }^{45} \mathrm{M}$. Berz and K. Makino. Higher order multivariate automatic differentiation and validated computation of remainder bounds. Transactions on Mathematics, 3,1:37-44, 2004 .

${ }^{46}$ M. Berz, K. Makino, and C. J. Johnstone. Propagation of a large-emittance muon beam through a straight, quadrupole-based precooling channel. In Neutrino Factories and Superbeams, volume 721, page 413. AIP Conference Proceedings, 2004.

${ }^{47}$ M. M. Alsharo'a et al. Recent progress in neutrino factory and muon collider research within the muon collaboration. Physical Review ST-AB, 6:081001, 2003.

${ }^{48}$ M. Berz. Cauchy theory on Levi-Civita fields. Contemporary Mathematics, 319:39-52, 2003.

${ }^{49} \mathrm{M}$. Berz and K. Makino. Constructive generation and verification of Lyapunov functions around fixed points of nonlinear dynamical systems. International Journal of Computer Research, 12,2:235-244, 2003.

${ }^{50} \mathrm{~K}$. Makino and M. Berz. Taylor models and other validated functional inclusion methods. International Journal of Pure and Applied Mathematics, 6,3:239-316, 2003. 
${ }^{51}$ J. Hoefkens, M. Berz, and K. Makino. Computing validated solutions of implicit differential equations. Advances in Computational Mathematics, 19:231-253, 2003.

${ }^{52}$ K. Makino and M. Berz. Verified global optimization with Taylor model methods. International Journal of Computer Research, 12,2:245-252, 2003.

${ }^{53} \mathrm{~K}$. Shamseddine and M. Berz. Measure theory and integration on the Levi-Civita field. Contemporary Mathematics, 319:369-387, 2003.

${ }^{54}$ J. Hoefkens, M. Berz, and K. Makino. Controlling the wrapping effect in the solution of ODEs of asteroids. Reliable Computing, 9(1):21-41, 2003.

${ }^{55}$ A. Geraci, T. Barlow, M. Portillo, J. Nolen, K. Shepard, M. Berz, and K. Makino. Highorder maps with acceleration for optimization of electrostatic and radio-frequency ion-optical elements. Review of Scientific Instruments, 73,9:3174-3180, 2002.

${ }^{56}$ J. Hoefkens, M. Berz, and K. Makino. Efficient high-order methods for ODEs and DAEs. In G. Corliss, C. Faure, A. Griewank, L. Hascoët, and U. Naumann, editors, Automatic Differentiation of Algorithms from Simulation to Optimization, pages 343348. Springer, 2002.

${ }^{57}$ K. Makino and M. Berz. Solenoid elements in COSY INFINITY. IOP CP, 175:219$228,2002$.

${ }^{58} \mathrm{~K}$. Makino and M. Berz. High order flows around fixed points and verification of stability of local dynamics with applications to particle accelerators. Complexity, 2002.

${ }^{59}$ L. M. Chapin, J. Hoefkens, and M. Berz. The COSY language independent architecture: Porting COSY source files. IOP CP, 175:37-45, 2002.

${ }^{60} \mathrm{~K}$. Shamseddine and M. Berz. Intermediate values and inverse functions on nonArchimedean fields. International Journal of Mathematics and Mathematical Sciences, 30:165-176, 2002.

${ }^{61}$ K. Makino and M. Berz. New applications of Taylor model methods. In G. Corliss, C. Faure, A. Griewank, L. Hascoët, and U. Naumann, editors, Automatic Differentiation of Algorithms from Simulation to Optimization, pages 359-364. Springer, 2002.

${ }^{62}$ C. O. Maidana, M. Berz, and K. Makino. Muon beam ring cooler simulations using COSY INFINITY. IOP CP, 175:211-218, 2002.

${ }^{63} \mathrm{~J}$. Hoefkens and M. Berz. Verification of invertibility of complicated functions over large domains. Reliable Computing, 8,1:1-16, 2002.

${ }^{64}$ M. L. Shashikant, M. Berz, and B. Erdélyi. COSY INFINITY's EXPO symplectic tracking for LHC. IOP CP, 175:299-305, 2002.

${ }^{65} \mathrm{M}$. Berz. Towards a universal data type for scientific computing. In G. Corliss, C. Faure, A. Griewank, L. Hascoët, and U. Naumann, editors, Automatic Differentiation of Algorithms from Simulation to Optimization, pages 373-381. Springer, 2002.

${ }^{66}$ B. Erdélyi and M. Berz. Optimal symplectic approximation of Hamiltonian flows. Physical Review Letters, 87,11:114302, 2001.

${ }^{67} \mathrm{M}$. Berz and J. Hoefkens. Verified high-order inversion of functional dependencies and superconvergent interval Newton methods. Reliable Computing, 7(5):379-398, 2001. 
${ }^{68} \mathrm{M}$. Berz, B. Erdélyi, and K. Makino. Towards accurate simulation of fringe field effects. Nuclear Instruments and Methods A, 472,3:533-540, 2001.

${ }^{69}$ K. Makino and M. Berz. Higher order verified inclusions of multidimensional systems by Taylor models. Nonlinear Analysis, 47:3503-3514, 2001.

${ }^{70} \mathrm{~S}$. Ozaki et al. for the Muon Collaboration. Feasibility study-II of a muon-based neutrino source. Technical Report 52623, Muon Collider Collaboration, BNL, 2001.

${ }^{71}$ B. Erdélyi, J. Hoefkens, and M. Berz. Rigorous lower bounds for the domains of definition of extended generating functions. SIAM Journal on Applied Dynamical Systems, 2001.

${ }^{72}$ J. Hoefkens, L. Diening, M. Berz, and B. Erdélyi. The WebCOSY system for course management in distance education. Journal of Computers in Mathematics and Science Teaching, 20(3):307-321, 2001.

${ }^{73}$ J. Hoefkens, M. Berz, and K. Makino. Verified high-order integration of DAEs and higher-order ODEs. In W. Kraemer and J.W. v. Gudenburg, editors, Scientific Computing, Validated Numerics, Interval Methods, pages 281-292, Boston, 2001. Kluwer.

${ }^{74}$ M. Berz, K. Makino, and J. Hoefkens. Verified integration of dynamics in the solar system. Nonlinear Analysis, 47:179-190, 2001.

${ }^{75}$ M. Berz, B. Erdélyi, and K. Makino. Fringe field effects in small rings of large acceptance. Physical Review ST-AB, 3:124001, 2000.

${ }^{76}$ B. Erdélyi, J. Hoefkens, L. Diening, K. Makino, and M. Berz. The Michigan State University M.Sc. and Ph.D. on-line degree programs in beam physics. Quarterly Review of Distance Education, 1,4, 2000.

${ }^{77} \mathrm{~K}$. Shamseddine and M. Berz. The differential algebraic structure of the Levi-Civita field. International Journal of Applied Mathematics, 3,4:449-464, 2000.

${ }^{78} \mathrm{M}$. Berz and K. Makino. Preservation of canonical structure in nonplanar curvilinear coordinates. International Journal of Applied Mathematics, 3,4:401-419, 2000.

${ }^{79} \mathrm{~K}$. Makino and M. Berz. Perturbative equations of motion and differential operators in nonplanar curvilinear coordinates. International Journal of Applied Mathematics, $3,4: 421-440,2000$.

${ }^{80} \mathrm{M}$. Berz. Non-Archimedean analysis and rigorous computation. International Journal of Applied Mathematics, 2,5:889-930, 2000.

${ }^{81}$ M. Berz. Analytical and computational methods for the Levi-Civita fields. Proc. Sixth International Conference on Non-Archimedean Analysis, pages 21-34, 2000.

${ }^{82} \mathrm{~K}$. Shamseddine and M. Berz. Convergence on the Levi-Civita field and study of power series. Proc. Sixth International Conference on Non-Archimedean Analysis, pages 283-299, 2000.

${ }^{83}$ M. Berz, K. Makino, and B. Erdélyi. Fringe field effects in muon rings. AIP CP, 530:38-47, 2000.

${ }^{84} \mathrm{~K}$. Shamseddine and M. Berz. Power series on the Levi-Civita field. International Journal of Applied Mathematics, 2,5:931-952, 2000.

${ }^{85} \mathrm{~K}$. Makino and M. Berz. Effects of kinematic correction on the dynamics in muon rings. AIP $C P, 530: 217-227,2000$. 
${ }^{86} \mathrm{~K}$. Makino and M. Berz. Efficient control of the dependency problem based on Taylor model methods. Reliable Computing, 5(1):3-12, 1999.

${ }^{87}$ R. Degenhardt and M. Berz. High accuracy description of the fringe field in particle spectrographs. Nuclear Instruments and Methods, A427:151-156, 1999.

${ }^{88}$ M. Berz, B. Erdélyi, W. Wan, and K. Ng. Differential algebraic determination of highorder off-energy closed orbits, chromaticities, and momentum compactions. Nuclear Instruments and Methods, A427:310-314, 1999.

${ }^{89} \mathrm{M}$. Berz and K. Makino. New methods for high-dimensional verified quadrature. Reliable Computing, 5(1):13-22, 1999.

${ }^{90}$ W. Wan, C. Johnstone, J. Holt, M. Berz, K. Makino, and M. Lindemann. The influence of fringe fields on particle dynamics in the large hadron collider. Nuclear Instruments and Methods, A427:74-78, 1999.

${ }^{91} \mathrm{~K}$. Makino and M. Berz. COSY INFINITY version 8. Nuclear Instruments and Methods, A427:338-343, 1999.

${ }^{92} \mathrm{M}$. Berz and K. Makino. Verified integration of ODEs and flows using differential algebraic methods on high-order Taylor models. Reliable Computing, 4(4):361-369, 1998.

${ }^{93} \mathrm{~W}$. Wan and M. Berz. Design of a fifth order achromat. Nuclear Instruments and Methods, 423-1:1-6, 1998.

${ }^{94}$ M. Berz. Computational Differentiation, Entry in 'Encyclopedia of Computer Science and Technology'. Marcel Dekker, New York, 1998.

${ }^{95}$ M. Berz, B. Erdélyi, and J. Hoefkens. Experience with interactive remote graduate instruction in beam physics. Journal of Interactive Learning Research, 10(1):49-58, 1998.

${ }^{96}$ M. Berz. Higher Order Derivatives and Taylor Models, Entry in 'Encyclopedia of Optimization'. Kluwer, Boston, in print.

${ }^{97}$ M. Berz. Differential Algebraic Techniques, Entry in 'Handbook of Accelerator Physics and Engineering', M. Tigner and A. Chao (Eds.). World Scientific, New York, 1999.

${ }^{98}$ G. Hoffstätter and M. Berz. Symplectic scaling of transfer maps including fringe fields. Physical Review E, 54,4, 1996.

${ }^{99} \mathrm{M}$. Berz and G. Hoffstätter. Computation and application of Taylor polynomials with interval remainder bounds. Reliable Computing, 4(1):83-97, 1998.

${ }^{100}$ W. Wan and M. Berz. An analytical theory of arbitrary order achromats. Physical Review E, 54,3:2870, 1996.

${ }^{101}$ V. Balandin, M. Berz, and N. Golubeva. Algorithms for the treatment and analysis of spin dynamics in $\mathrm{SU}(2)$ and $\mathrm{SO}(3)$. AIP CP, 405:55-63, 1997.

${ }^{102}$ M. Berz. From Taylor series to Taylor models. AIP CP, 405:1-20, 1997.

${ }^{103} \mathrm{G}$. Hoffstätter and M. Berz. Rigorous lower bounds on the survival time in particle accelerators. Particle Accelerators, 54:193-202, 1996.

${ }^{104}$ V. Balandin, M. Berz, and N. Golubeva. Computation and analysis of spin dynamics. AIP CP, 391:276, 1996. 
${ }^{105}$ K. Makino and M. Berz. COSY INFINITY Version 7. AIP CP, 391:253, 1996.

${ }^{106} \mathrm{M}$. Berz. Differential algebras with remainder and rigorous proofs of long-term stability. AIP CP, 391:221, 1996.

${ }^{107}$ M. Berz, K. Makino, K. Shamseddine, G. Hoffstätter, and W. Wan. COSY INFINITY and its applications to nonlinear dynamics. In M. Berz, C. Bischof, G. Corliss, and A. Griewank, editors, Computational Differentiation: Techniques, Applications, and Tools, pages 363-365, Philadelphia, 1996. SIAM.

${ }^{108} \mathrm{~K}$. Makino and M. Berz. Remainder differential algebras and their applications. In M. Berz, C. Bischof, G. Corliss, and A. Griewank, editors, Computational Differentiation: Techniques, Applications, and Tools, pages 63-74, Philadelphia, 1996. SIAM.

${ }^{109}$ M. Berz. Calculus and numerics on Levi-Civita fields. In M. Berz, C. Bischof, G. Corliss, and A. Griewank, editors, Computational Differentiation: Techniques, Applications, and Tools, pages 19-35, Philadelphia, 1996. SIAM.

${ }^{110} \mathrm{~K}$. Shamseddine and M. Berz. Exception handling in derivative computation with non-Archimedean calculus. In M. Berz, C. Bischof, G. Corliss, and A. Griewank, editors, Computational Differentiation: Techniques, Applications, and Tools, pages 37-51, Philadelphia, 1996. SIAM.

${ }^{111} \mathrm{G}$. Hoffstätter and M. Berz. Methods of bounding long term stability in storage rings by estimating pseudo invariants of nonlinear motion. Particle Accelerators, 1996.

${ }^{112}$ M. Berz. Differential algebraic description and analysis of spin dynamics. AIP CP, 343, 1995.

${ }^{113} \mathrm{G}$. Hoffstätter and M. Berz. Accurate and fast computation of high-order fringe field maps via symplectic scaling. Nuclear Instruments and Methods, 363:124, 1995.

${ }^{114} \mathrm{~W}$. Wan and M. Berz. Beamlines without midplane symmetry for neutron and proton cancer therapy. Nuclear Instruments and Methods A, 363:142, 1995.

${ }^{115}$ M. Berz. Modern map methods for charged particle optics. Nuclear Instruments and Methods, 363:100, 1995.

${ }^{116}$ I. Ozaki, F. Kimura, and M. Berz. A new approach to higher-order sensitivity analysis for optimal mechanical design. Computational Mechanics, 16:223, 1994.

${ }^{117}$ W. Wan and M. Berz. Design of a fifth order achromat. Nuclear Instruments and Methods, 352, 1994.

${ }^{118}$ M. Berz and G. Hoffstätter. Exact bounds of the long term stability of weakly nonlinear systems applied to the design of large storage rings. Interval Computations, 2:68-89, 1994.

${ }^{119}$ M. Berz, K. Joh, J. A. Nolen, B. M. Sherrill, and A. F. Zeller. Reconstructive correction of aberrations in nuclear particle spectrographs. Physical Review C, 47,2:537, 1993.

${ }^{120} \mathrm{P}$. Snopok, M. Berz, and C. Johnstone. Calculating the nonlinear tune shifts with amplitude using measured data. In Proceedings 2007 Particle Accelerator Conference, 2007.

${ }^{121}$ P. Snopok, M. Berz, and C. Johnstone. A new lattice design for a $1.5 \mathrm{GeV} \mathrm{CoM}$ muon collider consistent with the Tevatron tunnel. In Proceedings 2007 Particle Accelerator Conference, 2007. 
${ }^{122}$ P. Snopok, M. Berz, and C. Johnstone. High-order effects and modeling of the Tevatron. In Proceedings 2006 European Particle Accelerator Conference, 2006.

${ }^{123}$ C. Johnstone, M. Berz, and K. Makino. Linear quadrupole cooling channel for a neutrino factory. In Proceedings 2005 Particle Accelerator Conference, 2005.

${ }^{124}$ P. Snopok, C. Johnstone, and M. Berz. Decoupling schemes for the Tevatron in the presence of skew quadrupole fields. In Proceedings 2005 Particle Accelerator Conference, 2005.

${ }^{125}$ P. Snopok, C. Johnstone, M. Berz, D. A. Ovsyannikov, and A. D. Ovsyannikov. Highorder simulation of a muon collider interaction region. In Proceedings, Stability and Control Processes 2005, pages 292-297, 2005.

${ }^{126}$ A. Poklonskiy, D. Neuffer, M. Berz, D. A. Ovsyannikov, and A. D. Ovsyannikov. Optimization of the buncher and phase rotator for a neutrino factory. In Proceedings, Stability and Control Processes 2005, pages 282-291, 2005.

${ }^{127}$ A. A. Poklonskiy, D. A. Ovsyannikov, D. Neuffer, and M. Berz. Exploring the bunching section of the neutrino factory. In Proceedings, Physics and Control 2005, pages 272$277,2005$.

${ }^{128}$ P. Snopok, D. A. Ovsyannikov, A. D. Ovsyannikov, C. Johnstone, and M. Berz. Muon collider interaction region simulation and optimization. In Proceedings, Physics and Control 2005, pages 278-281, 2005.

${ }^{129}$ C. J. Johnstone, M. Berz, D. Errede, and K. Makino. Optimization and beam control in large-emittance accelerators: Neutrino factories. In 2003 International Conference Physics and Control, pages 964-973. IEEE, 2003.

${ }^{130} \mathrm{M}$. Berz and K. Makino. Recent advances in differential algebraic methods. In Proc. of the APS/DPF/DPB Summer Study on the Future of Particle Physics (Snowmass 2001), number T509, 2002. http://www.slac.stanford.edu/econf/C010630/papers/T509.PDF.

${ }^{131} \mathrm{M}$. Berz and K. Makino. Normal form methods and optimization for nonlinear properties of cooling channels - part I. In Proc. of the APS/DPF/DPB Summer Study on the Future of Particle Physics (Snowmass 2001), number T504, 2002. http://www.slac.stanford.edu/econf/C010630/papers/T504.PDF.

${ }^{132} \mathrm{M}$. Berz and K. Makino. Normal form methods and optimization for nonlinear properties of cooling channels - part II. In Proc. of the APS/DPF/DPB Summer Study on the Future of Particle Physics (Snowmass 2001), number T701, 2002. http://www.slac.stanford.edu/econf/C010630/papers/T701.PDF.

${ }^{133}$ K. Makino, D. Errede, and M. Berz. Cooling channel simulations based on map methods. In Proc. of the APS/DPF/DPB Summer Study on the Future of Particle Physics (Snowmass 2001), number T702, 2002. http://www.slac.stanford.edu/econf/C010630/papers/T702.PDF.

${ }^{134} \mathrm{~K}$. Makino and M. Berz. Recent applications of COSY to nonlinear beam dynamics problems. In Proc. of the APS/DPF/DPB Summer Study on the Future of Particle Physics (Snowmass 2001), number T510, 2002. http://www.slac.stanford.edu/econf/C010630/papers/T510.PDF.

${ }^{135}$ B. Erdélyi, J. Hoefkens, M. Berz, and K. Makino. Distance education in beam physics. In N. Nistor, S. English, and S. Wheeler, editors, Towards the Virtual University International Online Learning Perspectives, 2001. 
${ }^{136}$ B. Erdélyi, J. Hoefkens, L. Diening, K. Makino, and M. Berz. The Michigan State University M.Sc. and Ph.D. on-line degree programs in beam physics. Proceedings, 2000 International Conference on Distance Education, 2000.

${ }^{137}$ K. Makino, B. Erdélyi, and M. Berz. Magnet fringe fields, nonlinear effects, and compensation in large acceptance rings. In 2001 Particle Accelerator Conference. IEEE, 2001. See also http://pac2001-ed.fnal.gov/, paper ID WOPB008.

${ }^{138}$ K. Makino and M. Berz. Map-based muon cooling channel simulations with COSY INFINITY. In B. King, editor, Proc. of 6-Month Feasibility Study on High Energy Muon Colliders, 2001. http://pubweb.bnl.gov/people/bking/mucoll/index.html.

${ }^{139}$ K. Makino and M. Berz. Properties of the Taylor model method for rigorous estimates. In Beam Dynamics Optimization 98, pages 86-90. Saint-Petersburg State University, 2002.

${ }^{140} \mathrm{M}$. Berz and K. Makino. Rigorous integration of maps. In Beam Dynamics Optimization 98, pages 61-66. Saint-Petersburg State University, 2002.

${ }^{141} \mathrm{M}$. Berz, B. Erdélyi, and J. Hoefkens. Large scale remote graduate instruction in beam physics. In Proceedings, WEBNET $199 \%$.

${ }^{142}$ K. Shamseddine and M. Berz. The non-Archimedean field $\mathcal{R}$, overview and applications. In Proceedings of the International Conference on Scientific Computations, Beirut, Lebanon, 1999.

${ }^{143}$ K. Shamseddine and M. Berz. Power series on the non-Archimedean field $\mathcal{R}$. In Proceedings of the International Conference on Scientific Computations, Beirut, Lebanon, 1999.

${ }^{144}$ M. Berz, B. Erdélyi, and J. Hoefkens. Distance education in graduate physics. In Proceedings, 1997 Conference on the Internet as a Vehicle for Teaching, Bucharest, 1997.

${ }^{145} \mathrm{~K}$. Shamseddine and M. Berz. Non-Archimedean structures as differentiation tools. In Proceedings, Second LAAS International Conference on Computer Simulations, pages 471-480, 1997.

${ }^{146}$ K. Makino and M. Berz. Arbitrary order aberrations for elements characterized by measured fields. In Optical Science, Engineering and Instrumentation '97, volume 3155, pages 221-227. SPIE, 1997.

${ }^{147}$ M. Berz and K. Makino. Arbitrary order maps, remainder terms, and long term stability in particle accelerators. In Optical Science, Engineering and Instrumentation '97, volume 3155, pages 186-192. SPIE, 1997.

${ }^{148}$ M. Berz. COSY INFINITY version 7. In 1997 Particle Accelerator Conference. APS, 1997.

${ }^{149}$ K. Makino. Rigorous integration of maps and long-term stability. In 1997 Particle Accelerator Conference, pages 1336-1340. APS, 1998.

${ }^{150} \mathrm{M}$. Berz. Rigorous long-term stability estimates through remainder differential algebras. In Proceedings, Fourth International Conference on Beam Dynamics and Optimization, 1996. 
${ }^{151} \mathrm{M}$. Berz. Infinitely small numbers and almost infinitely large accelerators, or: Automatic differentiation as non-Archimedean analysis. In 1993 Argonne Theory Institute Report, ANL/MCS-TM-183, Argonne National Laboratory, 1994.

${ }^{152}$ P. Snopok, C. Johnstone, and A. Garren. Recent progress in muon collider lattice design. Technical Report MSUHEP-070420, Department of Physics and Astronomy, Michigan State University, East Lansing, MI 48824, 2007.

${ }^{153}$ P. Snopok, M. Berz, and C. Johnstone. Calculation of nonlinear tune shift using beam position measurement results. Technical Report MSUHEP-070421, Department of Physics and Astronomy, Michigan State University, East Lansing, MI 48824, 2007.

${ }^{154} \mathrm{M}$. Berz and K. Makino. COSY INFINITY Version 9.0 programmer's manual. Technical Report MSUHEP-060803, Department of Physics and Astronomy, Michigan State University, East Lansing, MI 48824, 2006. See also http://cosyinfinity.org.

${ }^{155} \mathrm{M}$. Berz and K. Makino. COSY INFINITY Version 9.0 beam physics manual. Technical Report MSUHEP-060804, Department of Physics and Astronomy, Michigan State University, East Lansing, MI 48824, 2006. See also http://cosyinfinity.org.

${ }^{156}$ S. Manikonda, J. Nolen, M. Berz, K. Makino, and G.Weizman. Design of a superconducting quadrupole with elliptical acceptance and tunable higher order multipoles. Technical Report MSUHEP-060412, Department of Physics and Astronomy, Michigan State University, East Lansing, MI 48824, 2006.

${ }^{157} \mathrm{H}$. Geissel et al. Technical report on the design, construction, commissioning and operation of FAIR. Technical report, GSI Darmstadt, 2005.

${ }^{158}$ Pavel Snopok. A converter program for tevatron lattices from OptiM to COSY INFINITY. Technical Report MSUHEP-40909, Department of Physics and Astronomy, Michigan State University, East Lansing, MI 48824, 2004.

${ }^{159}$ K. Makino and M. Berz. The LDB, QDB, and QFB bounders. Technical Report MSUHEP-40617, Department of Physics and Astronomy, Michigan State University, East Lansing, MI 48824, June 2004.

${ }^{160}$ K. Makino and M. Berz. Implementation of Taylor model arithmetic. Technical Report MSUHEP-20511, Department of Physics and Astronomy, Michigan State University, East Lansing, MI 48824, May 2002.

${ }^{161} \mathrm{~K}$. Makino and M. Berz. The method of shrink wrapping for the validated solution of ODEs. Technical Report MSUHEP-20510, Department of Physics and Astronomy, Michigan State University, East Lansing, MI 48824, May 2002.

${ }^{162}$ M. Berz, J. Hoefkens, and K. Makino. COSY INFINITY Version 8.1 - programming manual. Technical Report MSUHEP-20703, Department of Physics and Astronomy, Michigan State University, East Lansing, MI 48824, $2002 . \quad$ see also http://cosy.pa.msu.edu.

${ }^{163}$ M. Berz and K. Makino. COSY INFINITY Version 8.1 - user's guide and reference manual. Technical Report MSUHEP-20704, Department of Physics and Astronomy, Michigan State University, East Lansing, MI 48824, 2002. see also http://cosy.pa.msu.edu.

${ }^{164}$ M. Berz K. Makino, C.J. Johnstone and B. Erdélyi. Nonlinear effects in the 20-GeV feasibility II muon storage ring. Technical Report MSUCL-1197, National Superconducting Cyclotron Laboratory, Michigan State University, East Lansing, MI 48824, 2001. 
${ }^{165}$ F. Zimmermann, C. Johnstone, M. Berz, B. Erdélyi, K. Makino, and W. Wan. Fringe fields and dynamic aperture in muon storage rings. Neutrino Factory/Muon Collider Notes MUC-NOTE-NEUTRINO-SRC-0095, Fermi National Accelerator Laboratory, 2000. see http://www-mucool.fnal.gov/notes/notes.html, also CERN SL 2000-011 A-P, and CERN NuFact Note 21, CERN.

${ }^{166}$ B. Erdélyi, M. Berz, and K. Makino. Detailed analysis of fringe field effects in the large hadron collider. Technical Report MSUCL-1129, National Superconducting Cyclotron Laboratory, Michigan State University, East Lansing, MI 48824, 1999.

${ }^{167}$ B. Erdélyi, M. Berz, W. Wan, and K.-Y. Ng. The differential algebraic and analytic calculation of momentum compaction and comparison with tracking codes and COSY INFINITY. Technical Report MSUCL-1096, National Superconducting Cyclotron Laboratory, Michigan State University, East Lansing, MI 48824, 1998.

${ }^{168}$ M. Berz. COSY INFINITY Version 8 reference manual. Technical Report MSUCL1088, National Superconducting Cyclotron Laboratory, Michigan State University, East Lansing, MI 48824, 1997. see also http://cosy.pa.msu.edu.

${ }^{169}$ S. Rolles and M. Berz. Problems about Levi-Civita fields. Technical report, Department of Physics, Michigan State University, 1997.

${ }^{170}$ M. Berz. COSY INFINITY Version 7 reference manual. Technical Report MSUCL-977, National Superconducting Cyclotron Laboratory, Michigan State University, East Lansing, MI 48824, 1995. see also http://cosy.pa.msu.edu.

${ }^{171}$ M. Berz. Analysis on a non-Archimedean extension of the real numbers. Lecture Notes, 1992 and 1995 Mathematics Summer Graduate Schools of the German National Merit Foundation. MSUCL-933, Department of Physics, Michigan State University, 1994.

${ }^{172}$ M. Berz. COSY INFINITY Version 6 reference manual. Technical Report MSUCL-869, National Superconducting Cyclotron Laboratory, Michigan State University, East Lansing, MI 48824, 1993. 